\title{
Large stem cell grafts could lead to erroneous interpretations of behavioral results?
}

To the editor:

The recent paper by Roy et al. ${ }^{1}$ presents highly interesting results that could represent a major advance in the area of stem cell-based brain repair. The results also illustrate that it can be difficult to evaluate functional effects of stem cell-derived transplants to the brain, and with this letter we want to highlight issues of experimental design that are relevant to the whole research field. In their groundbreaking report, the authors describe specific conditions for generating large numbers of dopaminergic neurons derived from human embryonic stem cells (hESC). Moreover, they describe dramatic behavioral effects of grafted hESC in a rat model of Parkinson disease and demonstrate survival of exceptionally large numbers of hESC-derived dopaminergic neurons in the transplants. These claims are unique and extremely important for the future development of restorative therapies in Parkinson disease. In addition, the study reveals specific problems that the whole research field faces in evaluating the functional efficacy of intracerebral transplants. The authors tested the grafted rats on three behavioral tasks: apomorphineinduced rotation, the adjusting step test and paw use in the cylinder test. In the latter test, there were no clear deficits in the hemiparkinsonian rats, and it was therefore not possible to study graft-induced effects. Results from both the adjusting step test and the apomorphineinduced rotation, however, indicated that the surviving dopamine neurons in the grafts were functional. In the adjusting step test, the authors unexpectedly reported that the deficits appeared in the limb ipsilateral to the nigrostriatal lesion and that graft-induced effects were observed on the same side. This is contrary to the literature, which consistently reports deficits in the limb contralateral to a nigrostriatal lesion ${ }^{2,3}$. Concerning drug-induced rotational behavior, the authors challenged the rats with apomorphine, which stimulates 'supersensitive' dopamine receptors in the striatum ipsilateral to the lesion. This induces turning behavior in a direction away from the side of the lesion. In the study by Roy et al., the rats completely stopped rotating contralaterally 4-6 weeks after transplantation. Taken together, the reported effect on rotational behavior was surprisingly extensive, and the onset of the effect was unexpectedly rapid after grafting 4,5 . It is apparent from earlier work, however, that apomorphineinduced rotational asymmetry in hemiparkinsonian rats can be mitigated not only by replacing the lost dopamine neurons, but also by damaging striatal output neurons ${ }^{6}$. In this paradigm, damage to striatal neurons and dopamine neuron replacement can have the same effect-namely, eliminating striatal neurons with supersensitive dopamine receptors ${ }^{7}$. The concern that damage of striatal neurons might underlie the reduction in apomorphineinduced rotation in the study by Roy et al. is further strengthened by the fact that the grafts were extremely large. Specifically, the authors write that the donor cells were dispersed on average over a radius of $1.6 \mathrm{~mm}$. Therefore, it can be estimated that the mean volume of the transplants was in the range of 15-20 $\mathrm{mm}^{3}$. Consequently, they would have filled out approximately half the space normally occupied by one rat striatum (around $33 \mathrm{~mm}^{3}$ in size $)^{8}$. An informative control experiment would have entailed grafting similar numbers of hESC-derived neurons lacking dopaminergic properties. Alternatively, selectively destroying the dopaminergic neurons in the graft by injection of the toxin 6-hydroxydopamine ${ }^{9}$ would have shown whether the functional recovery really was dependent upon dopamine released from the grafts.

The authors discuss continued proliferation of the grafted hESC-derived cells and rightfully consider that this issue has to be

Roy et al. reply:

We thank Christophersen and Brundin for their comments. They expressed two principal concerns: that our behavioral results might not have unequivocally established graft-dependent functional recovery and that our graft volumes might have been excessive relative to the striatal volume. We believe that each of these concerns addressed before any clinical trials can be considered. In light of the information we present, we believe that the issue of functional capacity of the transplants also has to be revisited. The data presented by Roy et al. do not unequivocally demonstrate that there is a dopamine-dependent recovery of function in the transplant recipients. Indeed, when designing and evaluating our own experiments, we note that overgrowth of transplanted tissue derived from proliferating stem cells can affect the behavior of the graft host in a confounding manner.

\section{Nicolaj Strøyer Christophersen \& Patrik Brundin \\ Neuronal Survival Unit, Wallenberg Neuroscience Center, Department of Experimental Medical Science, Lund University, BMC A10 22184 Lund, Sweden. e-mail:patrik.brundin@med.lu.se}

\section{COMPETING INTERESTS STATEMENT}

The authors declare competing financial interests (see the Nature Medicine website for details).

1. Roy, N.S. et al. Nat. Med. 12, 1259-1268 (2006).

2. Olsson, M., Nikkhah, G., Bentlage, C. \& Bjorklund, A. J. Neurosci. 15, 3863-3875 (1995).

3. Chang, J.W., Wachtel, S.R., Young, D. \& Kang, U.J. Neuroscience 88, 617-628 (1999).

4. Stromberg, I., Bygdeman, M., Goldstein, M., Seiger, A \& Olson, L. Neurosci. Lett. 71, 271-276 (1986).

5. Brown, V.J. \& Dunnett, S.B. Exp. Brain Res. 78, 214 218 (1989).

6. Marshall, J.F. \& Ungerstedt, U. Science 198, 62-64 (1977).

7. Brundin, P., Duan, W.-M. \& Sauer, H. in Functional Neural Transplantation (eds. Dunnett, S.B. \& Bjorklund, A.) Ch. 2 9-46 (Raven Press, New York 1994).

8. Anden, N.E., Fuxe, K., Hamberger, B. \& Hokfelt, T. Acta Physiol. Scand. 67, 306-312 (1966).

9. Dunnett, S.B., Hernandez, T.D., Summerfield, A., Jones, G.H. \& Arbuthnott, G. Exp. Brain Res. 71, 411-424 (1988). is based on a misreading of our paper, resulting in a misrepresentation of its data.

First, Christophersen and Brundin question the results of one of our functional assessments, the adjusting step test, incorrectly stating that our 6-hydroxydopamine (6-OHDA)-lesioned hemiparkinsonian rats suffered paw placement deficits ipsilateral to their lesions and grafts. 